\title{
Satisfacción de Pareja y Depresión: ¿Es la Función Reflexiva una Variable Interviniente?
}

\section{Couple Satisfaction and Depression: Is the Reflective Functioning an Intervening Variable?}

\author{
María José León \\ Universidad de Chile \\ Marcia Olhaberry y Cristóbal Hernández \\ Catalina Sieverson \\ Universidad de Chile
}

\begin{abstract}
En el marco de la salud mental individual y familiar, la relación entre satisfacción de pareja y sintomatología depresiva ha sido bien documentada. Una segunda generación de estudios se ha focalizado en encontrar variables que modulen esta relación; sin embargo, el rol de la función reflexiva (FR) en la relación entre estas 2 variables no ha sido sido estudiado. Este estudio exploró el efecto moderador de la función reflexiva (Reflective Functioning Scale) sobre la relación entre satisfacción de pareja (Relationship Assessment Scale) y sintomatología depresiva (Inventario para la Depresión de Beck I) en 50 parejas con hijos/as menores de 3 años, seleccionadas intencionadamente de 21 comunas de la Región Metropolitana de Chile. Un análisis de regresión multinivel reveló que la satisfacción de pareja tiene un efecto inverso sobre la sintomatología depresiva. También se observó un efecto de moderación de la FR en la relación entre estas 2 variables: personas con alta FR muestran una relación inversa de mayor intensidad entre satisfacción de pareja y depresión que las con baja FR. Se discuten las implicancias clínicas de estos resultados.
\end{abstract}

Palabras clave: función reflexiva, satisfacción de pareja, depresión, análisis multinivel, Chile

\begin{abstract}
Within the context of individual and family mental health, the relationship between couple satisfaction and depressive symptomatology has been well documented. A second generation of studies have focused on finding variables that could modulate this relationship; however, the role of the reflective functioning (RF) in the relationship between these 2 variables has not been examined. This study explored the moderating effect of reflective functioning (Reflective Functioning Scale) on the relationship between couple satisfaction (Relationship Assessment Scale) and depressive symptomatology (Beck Depression Inventory I) in a sample of 50 couples with children under 3 years old, purposively selected in 21 municipalities of the Metropolitan Region of Chile. A multilevel regression analysis revealed that couple satisfaction has an inverse effect on depressive symptomatology. The analysis also showed a moderating effect of $\mathrm{RF}$ on the relationship between these 2 variables: compared to people with low $\mathrm{RF}$, those with high RF display a more intense inverse correlation between couple satisfaction and depression. The clinical implications of these findings are discussed.
\end{abstract}

Keywords: reflective functioning, couple satisfaction, depression, multilevel regression, Chile

Las investigaciones actuales en salud mental de pareja han demostrado consistentemente cómo los síntomas depresivos de uno de los miembros impactan negativamente los niveles de satisfacción con la relación, la salud mental y el bienestar del otro, así como también constituyen un factor de riesgo para la salud mental de los hijos (Davies, Coe, Martin, Sturge-Apple \& Cummings, 2015; Tissot, Favez, Ghisletta, Frascarolo \& Despland, 2017).

La asociación entre satisfacción de pareja y síntomas depresivos ha sido bien estudiada y documentada (Beach, 2001; Fincham, Beach, Harold \& Osborne, 1997), existiendo una segunda generación de investigaciones focalizadas en identificar variables intervinientes que podrían modificar esta relación (Beach,

María José León y Catalina Sieverson, Facultad de Medicina, Universidad de Chile, Santiago, Chile; Marcia Olhaberry y Cristóbal Hernández, Escuela de Psicología, Pontificia Universidad Católica de Chile, Santiago, Chile.

El artículo es parte de la tesis de María José León para optar al Grado de Doctor en Psicoterapia de la Universidad de Chile.

La elaboración de este artículo contó con el apoyo otorgado por el Fondo Nacional de Desarrollo Científico y Tecnológico, a través del Proyecto FONDECYT de Iniciación N N 11140230, liderado por Marcia Olhaberry, y recibió apoyo del Fondo de Innovación para la Competitividad del Ministerio de Economía, Fomento y Turismo de Chile, a través de la Iniciativa Científica Milenio, Proyecto IS130005.

La correspondencia relativa a este artículo debe ser dirigida a Marcia Olhaberry, Escuela de Psicología, Pontificia Universidad Católica de Chile, Avda. Vicuña Mackenna 4890, Macul, Santiago, Chile. Email: mpolhabe@uc.cl 
2001). En esta línea, el estudio de los mecanismos subyacentes a la relación entre satisfacción de pareja y depresión ha cobrado mayor importancia, analizando algunas investigaciones la influencia del género y el neuroticismo (Davila, Karney, Hall \& Bradbury, 2003), del nivel de conflicto marital y el tiempo de relación (Kouros, Papp \& Cummings, 2008), así como también del estilo comunicacional y de apego (Heene, Buysse \& Van Oost, 2007).

En este contexto, el concepto de reflective-self function (mentalización o función reflexiva; en adelante FR), definido como "el observador interno de la vida mental" (Fonagy, Steele, Steele, Moran \& Higgitt, 1991, p. 202), es uno de los constructos relevantes en el desarrollo y organización del self y en la construcción de interacciones sociales, constituyéndose como un aspecto central del funcionamiento social humano y la salud mental (Fonagy, Gergely, Jurist \& Target, 2004). La FR se define como la capacidad de interpretar los comportamientos propios y de otros a la luz de estados mentales. Esto implica reconocer conscientemente que las experiencias dan lugar a creencias y emociones y que estas, junto a los deseos, dan origen a ciertos tipos de comportamiento (Fonagy et al., 2004). Tener un funcionamiento reflexivo requiere un esfuerzo mental y está asociado a la toma consciencia sobre lo que otros piensan y sienten, así como de los propios pensamientos, sentimientos y estados de ánimo. Es así como la FR facilita la construcción de relaciones con otros, principalmente con aquellos con quienes se está emocionalmente vinculado, como la pareja y los hijos (Allen, Fonagy \& Bateman, 2008).

Las investigaciones en FR muestran claramente dos grandes focos de estudio, uno que considera el apego, parentalidad y desarrollo infantil y otro que revisa y desarrolla intervenciones basadas en mentalización para mejorar el funcionamiento reflexivo (Ensink, Bégin, Normandin \& Fonagy, 2017; Steele \& Steele, 2008). Las investigaciones que consideran el primer foco han demostrado el rol de la FR, ya sea como moderador o mediador, en la relación entre distintas variables, como el estilo de apego de la madre y el estilo de apego del hijo (Slade, Grienenberger, Bernbach, Levy \& Locker, 2005), los síntomas depresivos de la madre y los comportamientos parentales (Wong, 2012) y las experiencias de maltrato en la infancia de los padres y el estilo de apego adolescente (Borelli, Compare, Snavely \& Decio, 2015). Los estudios que consideran el segundo foco, intervenciones psicoterapéuticas basadas en mentalización, comenzaron para el tratamiento de trastornos de personalidad limítrofe (Bateman \& Fonagy, 2008, 2013), ampliándose luego a otras problemáticas, como, por ejemplo, intervenciones dirigidas a madres en prisión y sus hijos (Sleed, Baradon \& Fonagy, 2013) y madres con abuso de sustancias (Suchman, Decoste, Rosenberger \& McMahon, 2012), entre otros.

Por otra parte, la sintomatología depresiva se ha vinculado a dificultades en la capacidad de mentalizar, interfiriendo específicamente en la habilidad para hacer inferencias respecto de la afectividad de sí mismo y del otro. Esto ocurre a partir de la distorsión que provocan los estados emocionales propios de la depresión, los que tiñen la experiencia y generan un mayor foco en la propia vivencia que en la de los otros miembros de la familia (Belvederi Murri et al., 2017; Fischer-Kern et al., 2013; Ladegaard, Lysaker, Larsen \& Videbech, 2014; Mattern et al., 2015; Sethna, Murray \& Ramchandani, 2012; Uekermann et al., 2008). Sin embargo, esta relación varía y depende de la severidad y cronicidad de los síntomas: cuando la sintomatología depresiva es baja o moderada, esta no se relacionaría con la FR (Turner, Wittkowski \& Hare, 2008).

Tanto desde un punto de vista teórico como empírico se describe que la satisfacción con la relación de pareja es una de las variables que juega un rol importante en la calidad del funcionamiento familiar (Shapiro, Gottman \& Carrère, 2000). En relación a las etapas del ciclo vital, la literatura científica muestra que la satisfacción de la pareja puede disminuir significativamente con el nacimiento de los hijos y durante los primeros años de crianza (Castellano, Velotti, Crowell \& Zavattini, 2014; Christopher, Umemura, Mann, Jacobvitz \& Hazen, 2015). En este sentido, el nacimiento de un hijo puede gatillar una crisis al interior de la pareja, debido a la necesidad de reorganizar la relación, el funcionamiento familiar y el espacio personal a partir de su llegada, quedando en un segundo plano las necesidades personales y de la pareja (Cox \& Paley, 2003; Favez et al., 2012).

Adicionalmente, estudios reportan consistentemente una mayor presencia de depresión en personas en edad fértil y con posterioridad al nacimiento de un hijo, con mayor prevalencia en mujeres que en hombres. En efecto, las tasas de depresión reportadas entre el 2009-2010 en Chile muestran que un 27,9\% de mujeres y un $11 \%$ de hombres entre 25 y 44 años presentan sintomatología depresiva (Chile, Ministerio de Salud [MINSAL], 2011) y que la sintomatología depresiva posterior al parto está presente en alrededor del $40 \%$ de las mujeres (MINSAL, 2013) y en un 10,4\% de los hombres (Paulson \& Bazemore, 2010). 
Estudios sobre sexo y roles de género han mostrado que los roles sociales y las influencias culturales constituyen variables que podrían explicar la mayor prevalencia de la depresión en mujeres. En este sentido, la importancia que las mujeres otorgan a las relaciones sociales, a las tareas del hogar y al cuidado de niños pequeños explicaría la mayor presencia de sintomatología depresiva en ellas durante la crianza temprana de los hijos (Hyde, Mezulis \& Abramson, 2008; Piccinelli \& Wilkinson, 2000).

La depresión en hombres ha sido menos estudiada que en mujeres; sin embargo, los estudios muestran que la sintomatología depresiva posterior al nacimiento de un hijo causa efectos adversos en ellos, en la salud mental de la mujer y también en la satisfacción de pareja (Bielawska-Batorowicz \& Kossakowska-Petrycka, 2006; Goodman, 2008; Paulson \& Bazemore, 2010; Wee, Skouteris, Pier, Richardson \& Milgrom, 2011). En estrecha relación con la satisfacción de pareja, la presencia de sintomatología depresiva además aumenta el nivel de conflicto parental y disminuye el soporte y apoyo entre ambos miembros (Tissot et al., 2017).

En relación a las trayectorias de la satisfacción de pareja y la sintomatología depresiva, los estudios muestran diferencias según sexo. Fincham et al. (1997) encontraron que para los hombres las trayectorias causales emergen desde la depresión hacia la satisfacción marital, mientras que para las mujeres las trayectorias causales fueron inversas, desde la satisfacción hacia la depresión. En relación con los efectos cruzados entre hombres y mujeres, algunos estudios muestran que la depresión en las mujeres predice un descenso en la satisfacción marital en los hombres (Faulkner, Davey \& Davey, 2005). En la misma línea, Gabriel, Beach y Bodenmann (2010) encontraron que la satisfacción marital y los síntomas depresivos dependen del sexo, sugiriendo la necesidad de generar modelos de análisis e intervención que sean sensibles al sexo de los miembros de la pareja.

Si bien los estudios que revisan la satisfacción de pareja y la sintomatología depresiva reportan diferencias según el sexo, los pocos estudios que han analizado la FR en hombres y mujeres muestran que los promedios en esta variable son semejantes (Borelli, St John, Cho \& Suchman, 2016; Fonagy et al., 1991). Esto podría explicarse considerando que la FR es una capacidad más estable que la satisfacción de pareja y la sintomatología depresiva y que, a su vez, constituye un mecanismo interno que está a la base de otros procesos psicológicos, como la regulación del afecto y el establecimiento de relaciones sociales saludables (Fonagy et al., 2004; Slade, 2005).

Otro aspecto relevante en la satisfacción de pareja es la habilidad para el manejo de conflictos, mostrando la literatura que la presencia de estos no resulta necesariamente destructiva, sino que lo potencialmente destructor son los patrones relacionales disfuncionales, que aluden a conflictivas sostenidas en el tiempo (Tapia Villanueva, Poulsen, Armijo, Pereira \& Sotomayor, 2009). Gottman (1999) describió que las parejas que referían satisfacción en su relación eran aquellas capaces de discriminar qué dificultades podían ser efectivamente resueltas y que, además, eran capaces de desarrollar diálogos respetuosos y emocionalmente afectuosos al referirse a sus problemas recurrentes. De esta manera, es posible pensar que la presencia de un funcionamiento reflexivo, que facilita el diálogo a partir de la compresión de sí mismo y del otro, podría favorecer el afrontamiento adecuado de los conflictos y las emociones negativas (Benbassat \& Priel, 2012), contribuyendo también a identificar las circunstancias que facilitan o dificultan la relación de pareja y la crianza (Benbassat \& Priel, 2015).

La FR ha sido estudiada principalmente en madres y escasamente en padres, no existiendo literatura científica que evalúe su relación con la satisfacción de pareja. Como se mencionó anteriormente, los vínculos que esboza la literatura son indirectos y fundamentalmente enfocados en el estudio de la FR y el desarrollo infantil, la salud mental y el funcionamiento parental. Sin embargo, a partir de la definición del constructo y de las investigaciones existentes entre padres/madres e hijos, es posible suponer que la FR puede cumplir un rol en la relación entre la satisfacción de pareja y la depresión parental, requiriéndose evidencia empírica para el testeo de este planteamiento.

Si bien la FR ha sido ampliamente estudiada en la última década, tanto para el desarrollo de modelos comprensivos en salud mental como para el diseño y evaluación de intervenciones psicoterapéuticas, la relación entre FR, satisfacción de pareja y síntomas depresivos parentales no ha sido empíricamente estudiada. A partir de los antecedentes anteriormente expuestos, este estudio tuvo como objetivo explorar la relación entre la satisfacción de pareja, la sintomatología depresiva y el nivel de la FR de parejas con hijos entre 12 y 36 meses. De acuerdo al objetivo planteado, se evaluó: (a) el rol del sexo en la relación entre la satisfacción de pareja y la sintomatología depresiva y (b) el rol de la FR en la relación entre la satisfacción de pareja y la sintomatología depresiva. Se esperaba una asociación inversa entre FR, sintomatología depresiva y nivel de satisfacción de pareja. Asimismo, y dada la escasez de literatura sobre el tema, la direccionalidad 
de las interacciones no fue hipotetizada. Se realizó un análisis exploratorio sobre el rol interviniente del sexo y de la FR en la relación entre satisfacción de pareja y sintomatología depresiva.

\section{Método}

En este estudio se utilizó una metodología cuantitativa, de diseño no experimental, transversal y correlacional.

\section{Participantes}

La muestra de este estudio fueron 50 hombres y 50 mujeres, en relación actual de pareja, con hijos e hijas en edad temprana, contactados a través de servicios de salud. Las parejas participantes residían en 21 comunas de la Región Metropolitana de Chile.

El muestreo fue intencionado y los criterios de inclusión considerados en el estudio fueron los siguientes: ser mayor de 18 años, en relación actual de pareja, con al menos un hijo entre 12 y 36 meses de edad con alguna de las siguientes dificultades socioemocionales: dificultad en la regulación conductual o emocional, dificultades en la regulación del sueño, llanto y/o la alimentación, dificultades en la relación con uno o ambos padres, evaluadas inicialmente por los servicios de salud derivantes. Posteriormente, las dificultades socioemocionales fueron evaluadas a través del cuestionario de auto-reporte para cuidadores ASQ-SE (Squires, Bricker \& Twombly, 2002) con la plantilla correspondiente a la edad de cada niño/a.

Los criterios de exclusión al estudio fueron: presencia de discapacidad intelectual, psicosis o daño orgánico, presencia de dependencia o abuso de sustancias en los adultos, presencia de abuso sexual y/o violencia física infantil actual en la familia y enfermedades médicas en el niño que expliquen sus dificultades socioemocionales, evaluadas por los servicios de salud derivantes.

Los participantes fueron derivados por profesionales de centros de salud, con atención directa a niños entre 12 y 36 meses y sus padres o fueron referidos por los mismos participantes del estudio.

Las mujeres participantes se encontraban en un rango de edad entre 20 y 43 años de edad $(M=31,52$, $D E=4,84)$ y los hombres, entre 22 y 49 años de edad $(M=33,58, D E=5,83)$. El promedio de hijos de las mujeres fue $1,72(D E=0,86)$ y de los hombres, $1,57(D E=0,71)$, teniendo como mínimo un hijo y máximo cuatro y habiendo algunos miembros de la pareja que tenían hijos de relaciones anteriores. Un $69,6 \%$ de las parejas se encontraba criando a su primer hijo.

El promedio de años de estudio fue semejante para mujeres y hombres (padres $M=15,18, D E=2,43$; madres $M=15,16, D E=2,39$ ), 37 mujeres y 41 hombres tenían un título técnico o universitario y 39 mujeres y 49 hombres tenían un trabajo actual remunerado. Respecto de la proporción de tratamiento psicológico actual, $10 \%$ de las mujeres y el $6 \%$ de los hombres se encontraba en tratamiento psicológico al momento de la evaluación.

\section{Instrumentos}

Ficha de antecedentes. Se usó para caracterizar sociodemográficamente al grupo familiar. Este instrumento contiene preguntas breves orientadas a obtener información sociodemográfica: nivel educacional, actividad actual y número de hijos. También incluye preguntas sobre el historial de tratamientos psicológicos y psiquiátricos.

Parent Development Interview - Revised Short Version (PDI-R; Slade, Aber, Berger, Bresgi \& Kaplan, 2012). Se utilizó para evaluar la FR. Este instrumento es una entrevista semi-estructurada individual dirigida a padres y madres de niños entre tres meses y 14 años, que evalúa narrativas derivadas de la relación actual y específica con un hijo. Las preguntas están dirigidas a promover la descripción que hacen de su hijo, de la relación que tienen con él, la descripción de ellos mismos como padre/madre y respecto de cómo las propias experiencias con sus padres impactan en su forma actual de ser padre/madre. La entrevista tiene una duración aproximada de una hora, es grabada en audio para luego transcribirse completamente y ser posteriormente analizada y codificada. Contiene 29 preguntas, 15 de ellas de demanda (e.g.: ¿Cómo le parece que su relación con (su hijo/a) está afectando el desarrollo de la personalidad de (su hijo/a)?) y 14 de permiso (e.g.: ¿Puede describirse como padre/madre?). Las preguntas de demanda, en comparación a las de permiso, son aquellas que explícitamente demandan el uso de la FR y son aquellas que se codifican para evaluar esta competencia (Fonagy, Target, Steele \& Steele, 1998). 
Las entrevistas fueron transcritas y posteriormente codificadas con el Adeendum Reflective Functioning Scale (RF Scale; Fonagy et al., 1998), manual especialmente adaptado para la codificación de este instrumento (Slade, Bernbach, Grienenberger, Levy \& Locker, 2005; Slade et al., 2012). La escala consta de 11 puntos que van de -1 (FR negativa) a 9 (FR completa o excepcional). Puntajes bajo 3 indican una pobre capacidad reflexiva, es decir, en su discurso predomina el escaso uso de estados mentales, o evitación de ellos, en ocasiones con narrativas egocéntricas o con muestras de distorsión. Puntajes entre 3 y 4 puntos muestran un nivel bajo de funcionamiento reflexivo, es decir, existe presencia de estados mentales en la narrativa, pero con una baja capacidad reflexiva, mientras que puntajes sobre 5 muestran una clara y evidente capacidad de reflexionar sobre estados mentales (Slade, Bernbach et al., 2005).

Dos codificadores ciegos al resultado de los participantes en las demás variables de estudio, entrenados y certificados como confiables, codificaron el $25 \%$ de las entrevistas en conjunto. Se evaluó el coeficiente de Kappa y el coeficiente de correlación de intra-clase (CCI) para calcular la confiabilidad inter-jueces, mostrando ambos buenos puntajes, $\kappa=0,76, p<0,001$, CCI $=0,89$.

Relationship Assessment Scale (RAS; Hendrick, 1988). Evalúa la satisfacción global con la relación de pareja. El instrumento es un cuestionario de auto-reporte que consta de siete ítems (e.g.: ¿ De qué manera considera usted que su esposa/o satisface sus necesidades?) evaluados en una escala Likert de cinco puntos, desde 1 (pobremente) a 5 (extremadamente bien); a mayor puntaje mayor satisfacción en la relación de pareja. Un estudio chileno reporta un alfa de Cronbach de 0,71 para este instrumento (Rivera, Cruz \& Muñoz, 2011); en el presente estudio se obtuvo un valor alfa de Cronbach de 0,92.

Inventario Para la Depresión de Beck I (BDI-I; Beck, Ward, Mendelson, Mock \& Erbaugh, 1961). Evalúa sintomatología depresiva en los adultos. El instrumento es un cuestionario de auto-reporte que consta de 21 ítems y con un puntaje teórico total que varía entre 0 y 63 , el que permite definir cuatro categorías de depresión, según rangos de puntajes: mínima o no deprimida (0-9 puntos), leve (10-18), moderada (19-29) y severa (30-63). Cada ítem o pregunta ofrece cuatro opciones de respuesta con puntajes entre 0 (e.g.: no me siento triste) y 3 (e.g.: ya no puedo soportar esta pena). En la versión chilena, actualmente en proceso de validación, se reporta un coeficiente alfa de Cronbach de 0,86 en muestra clínica (Valdés et al., 2017). En el presente estudio se obtuvo un alfa de Cronbach de 0,84.

\section{Procedimiento}

El estudio cuenta con certificación de los comités de ética institucionales de la Pontificia Universidad Católica de Chile, Universidad de Chile y de la Comisión Nacional de Investigación Científica y Tecnológica. Las mediciones fueron realizadas entre los años 2015 y 2016 por psicólogos que participaron exclusivamente en la investigación y evaluaron de manera individual a los participantes con los instrumentos mencionados.

El derivante, médico o psicólogo en su mayoría, al pesquisar alguna dificultad socioemocional en el niño le explica al padre o a la madre la existencia de un proyecto de investigación de la Pontificia Universidad Católica de Chile, el cual evalúa y entrega apoyo psicológico para familias con hijos entre 12 a 36 meses de edad, invitándoles a ser contactados para ser invitados a participar del proyecto. Luego de la derivación, los participantes son contactados telefónicamente por la coordinadora del proyecto de investigación, quien les explica en detalle el estudio, y, en caso de acceder a participar, se coordina la sesión de evaluación, la cual puede ser en el domicilio o en dependencias de la Escuela de Psicología de la Pontificia Universidad Católica de Chile, realizándose la mayoría en los domicilios de las familias. De las 58 parejas que accedieron a participar, ocho de ellas desertaron del estudio.

La evaluación fue realizada por un psicólogo clínico, quien lideraba la evaluación, acompañado de un estudiante de psicología. Los participantes comenzaban la evaluación con la lectura y firma de una carta de consentimiento informado, en la cual se explicitaba el objetivo del estudio, sus beneficios (de apoyo psicológico, no se entregaba beneficio económico) y riesgos, la confidencialidad en el manejo de los datos obtenidos y la voluntariedad de su participación. En este sentido, se explicitó a los participantes su libertad para dejar el estudio en cualquier momento, sin que esto afectara la atención recibida en el centro de salud al que regularmente asistían. Posteriormente se completaba la ficha de antecedentes sociodemográficos, luego respondían a los cuestionarios (BDI-I, RAS) y, finalmente, se llevaba a cabo la entrevista al padre y la madre de forma individual (PDI-R), la cual fue grabada en audio, para luego ser transcrita y codificada. 
Al finalizar la evaluación, se le entregaba a la familia una retroalimentación de la evaluación realizada e información psicoeducativa respecto de las dificultades que presentaban como padres y su hijo (e.g., manejo respetuoso de las pataletas, destete). Sumado a lo anterior, la totalidad de las familias participantes en este estudio recibieron una intervención breve, utilizando video-feedback, orientada a mejorar la calidad de las relaciones familiares y el desarrollo infantil.

\section{Análisis de Datos}

Los análisis se realizaron utilizando el software estadístico R (R Development Core Team, 2011).

En primer lugar, las parejas estudiadas fueron caracterizadas en función de sus antecedentes sociodemográficos y, posteriormente, de acuerdo a su FR, satisfacción de pareja y sintomatología depresiva. Luego, se estimó una matriz de correlaciones lineales de Pearson para evaluar las inter-relaciones entre las variables de estudio y las variables sociodemográficas (sexo, edad, años de educación, número de hijos, tener trabajo actual remunerado y tener tratamiento psicológico actual), tanto para padres como para madres.

Posteriormente, se procedió a realizar un modelo de regresión multinivel para dar cuenta de la naturaleza anidada de los datos en parejas (Kenny, Kashy \& Cook, 2006), utilizando el paquete nlme (Pinheiro, Bates, DebRoy, Sarkar \& R Core Team, 2017) de modelos lineales y no lineales mixtos para el software estadístico R. En primer lugar, se realizó un modelo nulo (sin predictores) para evaluar el grado en que la variabilidad de la sintomatología depresiva de los participantes es explicada por su pertenencia a la pareja. Dicho modelo permitió apreciar que un 13,2\% de la variabilidad de la sintomatología depresiva es explicada por la pareja (CCI: 0,132), lo que justifica la utilización de un modelo multinivel para así evitar la probabilidad de error tipo I asociada a ignorar la dependencia de los datos al grupo.

Para evaluar la influencia de la satisfacción de pareja y la FR en la sintomatología depresiva de las parejas, se realizó un modelo de regresión multinivel con un estimador REML (restricted maximum likelihood), dado que ofrece estimados de varianza más precisos para muestras pequeñas (Peugh, 2010). Para favorecer la interpretación de los datos y evitar los problemas de colinealidad, se centraron todos los predictores sobre su gran media (Shieh, 2011) y no sobre la media del grupo, para así evitar eliminar la variabilidad aportada por la pareja (Campbell \& Kashy, 2002; Kenny et al., 2006). Por otro lado, dado que los residuos del modelo no se distribuyeron normalmente, se transformó la variable dependiente, que presentaba una asimetría positiva, en base al logaritmo natural, para así asegurar una estimación más robusta. Con el objetivo de obtener estimados estandarizados, todas las variables fueron transformadas a puntaje $Z$.

\section{Resultados}

\section{Análisis Descriptivos de la Función Reflexiva, la Satisfacción de Pareja y la Sintomatología Depresiva}

Inicialmente, se evaluó la existencia de diferencias en las medias de madres y padres en la satisfacción de pareja (madres: $M=29,96, D E=4,84$; padres: $M=30,24, D E=5,94), t(98)=-0.297, p=0,767$, sintomatología depresiva (madres: $M=7,40, D E=6,06$; padres: $M=5,36, D E=5,94), t(98)=1,70, p=0,092$, y FR (madres: $M=3,64, D E=1,12$; padres: $M=3,56, D E=1,11), t(98)=0,445, p=0,657)$. Los resultados mostraron que no existen diferencias en las variables estudiadas entre padres y madres.

En relación a la sintomatología depresiva, se observó que el $66 \%$ de las mujeres y el $78 \%$ de los hombres presentaba sintomatología mínima o no deprimida/o, el $32 \%$ de las mujeres y el $20 \%$ de los hombres presentaban sintomatología leve y el $2 \%$ de las mujeres y el $2 \%$ de los hombres, síntomas moderados a severos.

En relación a la FR, se observó que el $24 \%$ de las madres y el $18 \%$ de los padres presentaban una clara y evidente capacidad de reflexionar sobre estados mentales. La mayor cantidad de parejas (64\% madres y $68 \%$ padres) muestra presencia de estados mentales en sus narrativas, pero con una baja capacidad reflexiva. Finalmente, el $12 \%$ de las madres y el $14 \%$ de los padres presentaban un nivel pobre de funcionamiento reflexivo, sin mostrar consciencia de los estados mentales.

\section{Análisis Correlacionales}

Solo se encontró una relación directa entre la FR y años de educación, razón por la que se consideró como variable control en los análisis posteriores. 
En relación a las principales variables del estudio, es posible observar una correlación inversa entre la satisfacción de pareja y la sintomatología depresiva (ver Tabla 1).

Tabla 1

Correlación entre las Variables Principales y Sociodemográficas del Estudio

\begin{tabular}{|c|c|c|c|c|c|c|c|c|c|}
\hline & 1 & 2 & 3 & 4 & 5 & 6 & 7 & 8 & 9 \\
\hline 1. Satisfacción pareja & 1 & & & & & & & & \\
\hline 2. Síntomas depresivos & $-0,474^{* *}$ & 1 & & & & & & & \\
\hline 3. Función reflexiva & 0,113 & 0,126 & 1 & & & & & & \\
\hline 4. Sexo & 0,030 & $-0,169$ & $-0,045$ & 1 & & & & & \\
\hline 5. Edad & $-0,067$ & 0,055 & 0,039 & 0,191 & 1 & & & & \\
\hline 7. Número de hijos & 0,105 & 0,036 & $-0,020$ & 0,000 & $0,535^{* *}$ & 1 & & & \\
\hline 6. Años de educación & $-0,059$ & 0,044 & $0,221^{*}$ & 0,025 & $-0,081$ & $-0,256^{*}$ & 1 & & \\
\hline 8. Trabajo actual & 0,027 & $-0,138$ & 0,132 & $0,268^{* *}$ & $0,265^{* *}$ & 0,118 & 0,023 & 1 & \\
\hline 9. Tratamiento psicológico actual & $-0,172$ & 0,098 & $-0,062$ & $-0,074$ & $0,229^{*}$ & 0,097 & $-0,043$ & 0,004 & 1 \\
\hline
\end{tabular}

Nota. Sexo: $1=$ mujer, $2=$ hombre, trabajo actual remunerado: $0=$ no tener trabajo, $1=$ tener trabajo, tratamiento psicológico actual: $0=$ no tener tratamiento actual, $1=$ tener tratamiento actual.

$* p<0,05, * * p<0,01$

\section{Regresión Multinivel}

Las variables agregadas al nivel 1 fueron el sexo (-0,5 mujer, 0,5 hombre), años de escolaridad del participante como variable de control, satisfacción con la relación de pareja y FR. Siguiendo la notación de Preacher, Curran y Bauer (2006), para los efectos de interacción se tomó como predictor focal la satisfacción de pareja, cuya relación con la sintomatología depresiva fue moderada por el sexo, por un lado, y la FR, por el otro. Al tratarse de datos diádicos (dos unidades por grupo), se dejó variar únicamente el intercepto según la variable de nivel 2 (pareja), ya que es necesario tener más unidades por grupo que efectos aleatorios en el modelo (Kenny et al., 2006).

Para estimar el modelo inicial se tomó la variable FR en su escala continua, mientras que en futuros análisis de moderación se dicotomizó en base a una desviación estándar por sobre y por debajo de la media.

El modelo propuesto puede resumirse en la ecuación de nivel 1:

$$
\begin{aligned}
\mathrm{BDI}_{\mathrm{ij}}= & \beta_{0 \mathrm{j}}+\beta_{1}(\text { Sexo })+\beta_{2}(\text { Años de escolaridad })+\beta_{3}(\text { Satisfacción de pareja })+\beta_{4}(\text { Función reflexiva }) \\
& +\beta_{5}(\text { Satisfacción de parejax Sexo })+\beta_{6}(\text { Satisfacción de pareja x Funcion reflexiva })+\mathrm{r}_{\mathrm{ij}}
\end{aligned}
$$

y en la siguiente ecuación de nivel 2:

$$
\beta_{0 j}=\gamma_{00}+\mu_{0 j}
$$

donde $\beta_{0 j}$ corresponde al intercepto del nivel 1, mientras que $\beta_{1}$ a $\beta_{6}$ representan los coeficientes de regresión del mismo nivel más su error $r_{\mathrm{ij}}$.

Por otro lado, en la ecuación de nivel 2 se presenta la estimación del efecto aleatorio del intercepto de nivel 1, con su intercepto promedio general ajustado por grupo $\left(\gamma_{00}\right)$ más la variabilidad de los interceptos alrededor del modelo general $\left(\mu_{0 j}\right)$.

Es posible observar en la Tabla 2 que, ajustando por variabilidad aportada por la variable de nivel 2, la satisfacción de pareja fue un predictor inverso de la sintomatología depresiva, de manera que mientras mayor satisfacción de la pareja menor fueron los síntomas depresivos, mientras que el sexo, los años de escolaridad y la FR no predijeron la sintomatología depresiva.

Por otro lado, se encontró un efecto de moderación del sexo sobre la relación entre la satisfacción de pareja y la sintomatología depresiva, observándose una asociación más intensa entre satisfacción en la relación de pareja y la sintomatología depresiva en los hombres que en las mujeres (ver Figura 1). Asimismo, fue posible observar que la FR también ejerció un efecto de moderación en la relación entre la satisfacción de pareja y la sintomatología depresiva, observándose que cuando hay una alta FR es más intensa la relación entre la 
satisfacción en la relación de pareja y la sintomatología depresiva (ver Figura 2). Dichos efectos de interacción fueron graficados utilizando el paquete Ggplot2 (Wickham, 2002) para el software estadístico R.

Tabla 2

Regresión Multinivel de la Sintomatología Depresiva

\begin{tabular}{lcccrr}
\hline \multicolumn{1}{c}{ Efecto fijo } & Beta & $\begin{array}{c}\text { Error } \\
\text { estándar }\end{array}$ & $g l$ & \multicolumn{1}{c}{$t$} & \multicolumn{1}{c}{$p$} \\
\hline Intercepto & 0,027 & 0,088 & 49 & 0,312 & 0,756 \\
Sexo & $-0,161$ & 0,082 & 44 & $-1,954$ & 0,057 \\
Años de escolaridad & 0,000 & 0,089 & 44 & $-0,002$ & 0,999 \\
Satisfacción pareja & $-0,552$ & 0,089 & 44 & $-6,186$ & $<0,001$ \\
Funcion reflexiva & 0,101 & 0,091 & 44 & 1,108 & 0,274 \\
Satisfacción pareja * Sexo & $-0,197$ & 0,085 & 44 & $-2,320$ & 0,025 \\
Satisfacción pareja * FR & $-0,192$ & 0,094 & 44 & $-2,034$ & 0,048 \\
\hline \multicolumn{1}{c}{ Efecto aleatorio } & Varianza & Residual & & & \\
\hline Intercepto & 0,243 & 0,799 & & \\
\hline
\end{tabular}

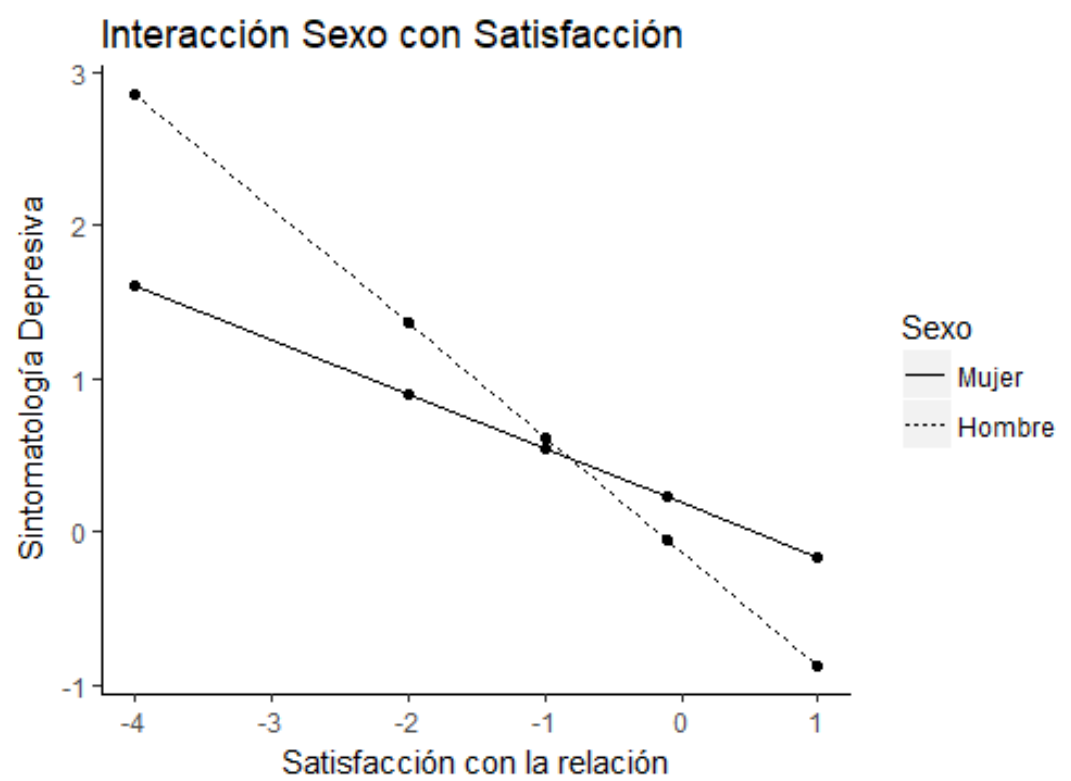

Figura 1. Efecto de moderación del sexo en la relación entre la satisfacción de pareja y la sintomatología depresiva.

Para evaluar más específicamente estos efectos de interacción, se realizó un análisis de pendientes simples (Hayes, 2013) en base al modelo multinivel antes propuesto, utilizando la librería "reghelper" para R (Hughes, 2017). En el caso del sexo, se calculó una pendiente individual para las mujeres y una pendiente individual para los hombres. En el caso de la FR, se calcularon las pendientes de la satisfacción de pareja para una desviación estándar hacia arriba y una desviación estándar por debajo de la función reflexiva.

Los resultados de los análisis para evaluar el rol del sexo en la relación entre la satisfacción de pareja y la depresión muestran que tanto en padres como en madres se observa una relación inversa entre satisfacción de pareja y depresión, pero en el caso de los padres esta relación presenta mayor intensidad, mostrando un efecto que duplica en tamaño al de las madres (ver Tabla 3). 


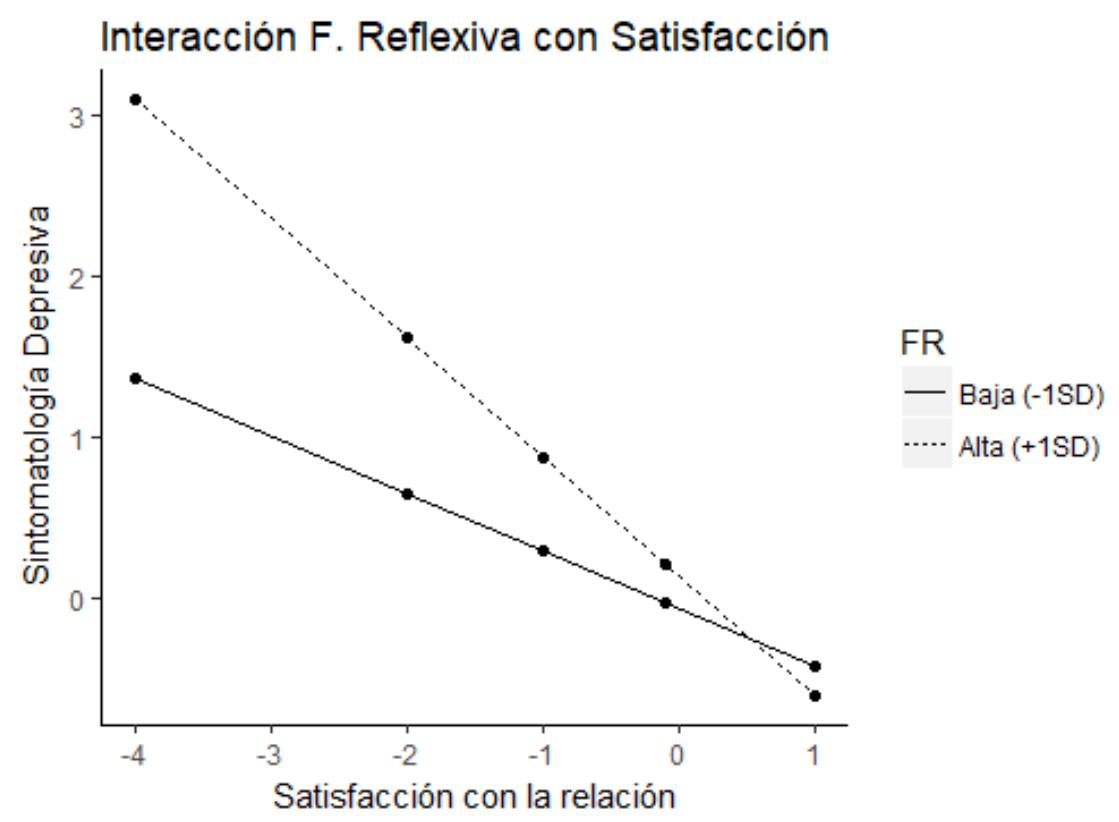

Figura 2. Efecto de moderación de la FR en la relación entre la satisfacción de pareja y la sintomatología depresiva.

Tabla 3

Análisis de Pendientes Simples por Sexo

\begin{tabular}{lccccc}
\hline \multicolumn{1}{c}{ Satisfacción pareja } & Beta & $\begin{array}{c}\text { Error } \\
\text { estándar }\end{array}$ & $g l$ & $t$ & $p$ \\
\hline Mujeres & $-0,355$ & 0,025 & 44 & $-3,06$ & 0,004 \\
Hombres & $-0,749$ & 0,025 & 44 & $-5,77$ & $<0,001$ \\
\hline
\end{tabular}

Nota. Variable dependiente: Sintomatología depresiva.

En los resultados de los análisis para evaluar el rol de la FR en la relación entre la satisfacción de pareja y la depresión es posible observar un efecto inverso de la satisfacción de pareja sobre la sintomatología depresiva, el que, sin embargo, resulta más robusto en el grupo con una alta FR. El grupo que presenta alta FR muestra una relación inversa de mayor intensidad entre satisfacción de pareja y depresión (ver Tabla 4). Adicionalmente, este grupo mostró un efecto del doble del tamaño que el grupo con una baja FR. Resulta importante notar que esta diferencia muestra una tendencia a amplificarse conforme aumentan las diferencias en la FR desde una desviación estándar a dos desviaciones estándar (Figura 2)

Tabla 4

Análisis de Pendientes Simples por FR

\begin{tabular}{|c|c|c|c|c|c|}
\hline Satisfacción pareja & Beta & $\begin{array}{c}\text { Error } \\
\text { estándar }\end{array}$ & $g l$ & $t$ & $p$ \\
\hline \multicolumn{6}{|c|}{ FR en base a la desviación estándar } \\
\hline $\mathrm{FR}(+1 D E)$ & $-0,744$ & 0,142 & 44 & $-5,23$ & $<0,001$ \\
\hline FR $(-1 D E)$ & $-0,359$ & 0,116 & 44 & $-3,10$ & 0,003 \\
\hline
\end{tabular}

Nota. Variable dependiente: Sintomatología depresiva. 
Finalmente, cabe destacar que para modelos multinivel es posible contar con dos estimados de varianza explicada, $R^{2}$ marginal, que describe la varianza explicada por los efectos fijos, y $R^{2}$ condicional, que describe la varianza explicada por los efectos fijos y aleatorios en su conjunto (Nakagawa \& Schieltzeth, 2013), los que fueron calculados utilizando el paquete piecewise SEM (Lefcheck, 2016) para R, que contiene una función para estimar aquellos valores en modelos lineales mixtos y modelos lineales mixtos generalizados. Se observó, entonces, que los efectos fijos por sí solos explicaron un 35\% de la varianza de la sintomatología depresiva $\left(R^{2}{ }_{\operatorname{GLMM}(m)}=0,349\right)$, mientras que los efectos fijos y aleatorios en su conjunto explicaron un $40 \%$ de la varianza de la misma $\left(R^{2} \operatorname{GLMM}(c)=0,396\right)$.

\section{Discusión}

Los resultados de este estudio proveen evidencia sobre la relación entre la satisfacción de pareja, la FR y la sintomatología depresiva en madres y padres, variables relevantes para la salud mental individual y de pareja en familias tempranas, que hasta la fecha no habían sido estudiadas en conjunto.

Respecto a los resultados descriptivos, es relevante mencionar que los promedios en la FR son más bajos que los obtenidos en investigaciones internacionales en padres y madres (Ensink, Bégin, Normandin \& Fonagy, 2016; Slade, Grienenberger et al., 2005). Los promedios en hombres y mujeres indican que sus relatos presentan estados mentales tales como feliz, seguro, triste, confiado, pero sin una evidente capacidad de reflexionar en torno a ellos. Esto es relevante, debido a que la literatura señala que niveles más altos de FR se relacionan con un estilo de apego seguro y, por el contrario, niveles más bajos se asocian a experiencias traumáticas sin elaborar y a patrones de apego inseguro (Borelli et al., 2015; Ensink et al., 2016).

En relación con la satisfacción de pareja, los promedios son semejantes a los obtenidos en otros estudios (Dinkel \& Balck, 2005), indicando niveles moderados a altos. En cuanto a la sintomatología depresiva, padres y madres muestran bajos niveles de depresión, con puntajes levemente mayores en el caso de las madres, lo que es coherente con la robusta literatura que señala la mayor prevalencia de depresión en mujeres, especialmente en edad fértil (MINSAL 2011, 2013; Paulson \& Bazemore, 2010).

Respecto de las hipótesis planteadas en este estudio, tal como se esperaba, la satisfacción de pareja fue un predictor significativo para la depresión, encontrándose que padres y madres menos satisfechos con su relación presentan una mayor sintomatología depresiva. Este resultado es similar a los reportados en investigaciones anteriores que dan cuenta de la relación entre estas variables (Beach, 2001; Fincham et al., 1997) y su impacto en la salud mental de la pareja y de los hijos (Davies et al., 2015; Tissot et al., 2017). En este sentido, los hallazgos de este estudio reafirman el valor de incorporar la variable satisfacción de pareja en los procesos de diagnóstico e intervención en padres y madres con hijos pequeños, ya que entrega información relevante sobre el subsistema parental.

Por otro lado, no se encontró una asociación entre FR y satisfacción de pareja y tampoco entre FR y síntomas depresivos. En relación a estos resultados, es importante destacar que el grupo estudiado corresponde a padres y madres consultantes por las dificultades en el desarrollo socioemocional de sus hijos, por lo que más allá del nivel de su funcionamiento reflexivo, forman parte de un sistema familiar que presenta síntomas infantiles y, por lo tanto, algún grado de pérdida de equilibrio y malestar parental. En este sentido, un adecuado nivel de FR no permitiría garantizar la ausencia de síntomas y malestar a nivel individual ni de pareja, sino más bien facilitaría la identificación del padecimiento subjetivo y su expresión en las escalas aplicadas para evaluar la satisfacción de pareja y la sintomatología depresiva. Resulta probable, también, que la asociación entre FR y satisfacción de pareja, así como entre FR y depresión, se observe en población general en sistemas familiares en equilibrio, dando cuenta de la capacidad de mentalizar como una herramienta para el enfrentamiento de la crianza temprana y el ajuste de pareja de manera adaptativa, sin la aparición de síntomas en los padres o en los niños. A partir de estos planteamientos, podrían también comprenderse los menores puntajes promedio en FR encontrados tanto en madres como en padres, en comparación con estudios internacionales, requiriéndose estudios en población general nacional para confirmar o refutar estos planteamientos.

Adicionalmente, pese a que la literatura muestra en ocasiones una asociación entre FR y sintomatología depresiva, esta relación depende de otros factores, como la severidad y cronicidad de los síntomas. Tal como muestra Turner et al. (2008), cuando la sintomatología depresiva es baja o moderada, esta no lograría teñir los procesos reflexivos, que posiblemente es lo que sucede en la muestra estudiada. 
En relación con la hipótesis del sexo como variable interviniente, el modelo utilizado confirma un efecto de moderación del sexo sobre la relación entre la satisfacción con la pareja y la sintomatología depresiva, tanto en hombres como mujeres. Madres y padres presentan una asociación inversa entre estas dos variables, pero, contrariamente a lo esperado, los padres presentan una relación de mayor intensidad, es decir, la baja satisfacción con la relación de pareja se asocia a mayores síntomas depresivos en los hombres que en las mujeres. Pese a que estudios previos muestran que uno de los factores que contribuye a explicar la depresión en mujeres es la mayor necesidad afiliativa (Hyde et al., 2008; Piccinelli \& Wilkinson, 2000), podría ocurrir que en mujeres con hijos/as pequeños/as con dificultades en el desarrollo socioemocional esto se presente de manera distinta. Es probable que la satisfacción de pareja juegue un rol menor en su sintomatología depresiva, preponderando la preocupación y el malestar asociado a su rol de cuidadora de un niño que requiere atención psicológica. En este sentido, las madres podrían estar focalizadas en la calidad de la relación materno-filial más que en la relación de pareja.

Este hecho, a su vez, pone en evidencia la necesidad de desarrollar modelos comprensivos que integren las diferencias según el sexo en esta variable, tanto para los diagnósticos como para la implementación de intervenciones psicoterapéuticas, diferenciando poblaciones clínicas y generales. Los hallazgos de este estudio se alinean con los planteamientos de Gabriel et al. (2010), quienes destacan la necesidad de generar modelos de análisis e intervención sensibles al sexo de los miembros de la pareja, siendo variables consideradas habitualmente en menor medida.

En relación a la principal hipótesis de este estudio, los resultados muestran que la FR cumple un rol moderador en la relación entre satisfacción de pareja y depresión. Específicamente, los resultados muestran un efecto inverso de la satisfacción de pareja sobre la sintomatología depresiva, el cual se intensifica en el grupo de sujetos con alta FR, es decir, aquellas personas que tienen una alta FR presentan una relación inversa más intensa entre satisfacción de pareja y síntomas depresivos.

Estos hallazgos resultan interesantes y podrían explicarse considerando que una mayor FR podría implicar mayores capacidades para la auto-observación y la identificación del propio bienestar y/o malestar. En este sentido, sería esperable que sujetos con una mayor capacidad reflexiva pudieran registrar y reportar con mayor certeza sus experiencias subjetivas y emocionales, lo que reflejaría su capacidad para reconocer las propias vivencias positivas y negativas e identificar el bienestar y malestar que estas generan.

Considerando, además, que el 69,6\% de los participantes son padres y madres de su primer/a hijo/a y, por lo tanto, están enfrentando un ajuste de pareja importante, cambios en su identidad y en sus roles asociados al inicio de la parentalidad. Los síntomas podrían indicar capacidad de contacto con el mundo interno en un momento de la vida que normativamente implica estrés, por lo que se esperaría que sujetos con mayor funcionamiento reflexivo muestren una mayor capacidad para identificarlo y expresarlo. En esta línea, incorporando, además, que se trata de padres que consultan por las dificultades socioemocionales de sus hijos/as, los síntomas podrían indicar un mayor grado de integración de la experiencia y el sufrimiento, ambos aspectos asociados a un mayor funcionamiento reflexivo. Lo anterior se observa también en los procesos habituales de psicoterapia, en que el contacto con el malestar emocional es un avance y la mejoría sintomática muestra trayectorias variables, experimentando muchas veces empeoramiento antes de una mejora significativa.

Otro aspecto interesante y novedoso de estos hallazgos es que la FR no necesariamente muestra una relación lineal con la sintomatología depresiva y con la satisfacción de pareja. Considerando que la FR es una capacidad que permite reflexionar acerca de aspectos individuales y/o relacionales asociados a procesos y estados psicológicos, la presencia de sintomatología depresiva podría dar cuenta de reconocimiento y elaboración de estados internos, propios de una adecuada capacidad reflexiva.

Adicionalmente, una adecuada FR no implica necesariamente que los conflictos de pareja serán resueltos, por esto, personas con un alto funcionamiento reflexivo pueden sentirse insatisfechas con su relación y presentar, además, una alta sintomatología depresiva, disponiendo de la capacidad de reconocer sus sentimientos y su malestar. Los resultados también indican que una adecuada FR no es siempre un factor protector directo para una buena relación de pareja y para la depresión, sino que favorece la capacidad de reconocer la propia subjetividad, la del otro y la mutua influencia, lo que no necesariamente resuelve el conflicto y reduce los síntomas.

Entre las limitaciones del estudio, es importante destacar el moderado tamaño muestral, lo que lleva a tomar los resultados con cautela, ya que no permite una variabilidad suficiente entre los participantes, especialmente respecto de la FR y la sintomatología depresiva (sería necesario contar con una mayor cantidad 
de sujetos con una alta FR y sintomatología depresiva). Por último, constituye también una limitación el que se trate de un estudio transversal. Probablemente el comportamiento de las variables y su asociación podría ser diferente en estudios longitudinales, como muestran algunas investigaciones que consideran depresión parental (Tissot et al., 2017).

En cuanto a futuros estudios que consideren estas variables, sería de gran valor clínico realizar investigación preventiva, considerando parejas que aún no se han convertido en padres y población general no consultante en salud mental. De esta forma se podrían abordar las dificultades en la relación y la sintomatología clínica sin la exigencia y las demandas asociadas a la parentalidad. Esto permitiría una mejor preparación en la pareja para la llegada de los hijos y el afrontamiento de los desafíos de la crianza, así como también reducir el impacto negativo de las tensiones parentales y la depresión en el desarrollo y la salud mental infantil. Futuros estudios también podrían incluir variables infantiles, lo que permitiría analizar la contribución de la FR en el desarrollo y la salud mental del niño y testear la hipótesis comprensiva que se propone para entender qué explica que padres con baja satisfacción y alta FR presenten alta sintomatología depresiva.

Respecto a las implicancias clínicas del estudio, resulta relevante mencionar la importancia de incluir en los diagnósticos y las intervenciones tempranas variables asociadas a la díada parental, como la satisfacción de pareja, considerando, además, las diferencias entre padres y madres. Un gran número de investigaciones focalizadas en este período ha estudiado la sintomatología depresiva parental, por sus efectos negativos en el desarrollo y la salud mental familiar e infantil, pero muy pocas han incluido la satisfacción de pareja y las diferencias entre padres y madres para comprenderla.

Sumado a lo anterior, el hecho de incluir variables de mayor estabilidad, como la FR, en la comprensión de la relación entre la satisfacción con la relación de pareja y los síntomas depresivos permite distinguir elementos subyacentes a la sintomatología que pueden constituir recursos para la intervención o dar cuenta de la necesidad de intervenciones a largo plazo.

En conclusión, este estudio confirma la relación que existe entre la satisfacción de pareja y la sintomatología depresiva, que ya ha sido demostrada de manera consistente y agrega la FR como un aspecto de mayor estabilidad en el funcionamiento para la comprensión de esta relación, que a su vez se asocia estrechamente a la salud mental.

\section{Referencias}

Allen, J. G., Fonagy, P. \& Bateman, A. W. (2008). Mentalizing in clinical practice. Arlington, VA: American Psychiatric Publishing.

Bateman, A. \& Fonagy, P. (2008). 8-year follow-up of patients treated for borderline personality disorder: Mentalization-based treatment versus treatment as usual. The American Journal of Psychiatry, 165, 631-638. https://doi.org/10.1176/appi.ajp.2007.07040636

Bateman, A. \& Fonagy, P. (2013). Mentalization-based treatment. Psychoanalytic Inquiry, 33, 595-613. https://doi.org/10.1080/07351690.2013.835170

Beach, S. R. H. (Ed). (2001). Marital and family processes in depression: A scientific foundation for clinical practice. Washington, DC: American Psychological Association.

Beck, A. T., Ward, C. H., Mendelson, M., Mock, J. \& Erbaugh, J. (1961). An inventory for measuring depression. Archives of General Psychiatry, 4, 561-571. https://doi.org/10.1001/archpsyc.1961.01710120031004

Belvederi Murri, M., Ferrigno, G., Penati, S., Muzio, C., Piccinini, G., Innamorati, M. ... Amore, M. (2017). Mentalization and depressive symptoms in a clinical sample of adolescents and young adults. Child and Adolescent Mental Health, 22, 69-76. https://doi.org/10.1111/camh.12195

Benbassat, N. \& Priel, B. (2012). Parenting and adolescent adjustment: The role of parental reflective function. Journal of Adolescence, 35, 163-174. https://doi.org/10.1016/j.adolescence.2011.03.004.

Benbassat, N. \& Priel, B. (2015). Why is fathers' reflective function important? Psychoanalytic Psychology, 32, 1-22. https://doi.org/10.1037/a0038022

Bielawska-Batorowicz, E. \& Kossakowska-Petrycka, K. (2006). Depressive mood in men after the birth of their offspring in relation to a partner's depression, social support, fathers' personality and prenatal expectations. Journal of Reproductive and Infant Psychology, 24, 21-29. https://doi.org/10.1080/02646830500475179

Borelli, J. L., Compare, A., Snavely, J. E. \& Decio, V. (2015). Reflective functioning moderates the association between perceptions of parental neglect and attachment in adolescence. Psychoanalytic Psychology, 32, 23-35. https://doi.org/10.1037/a0037858

Borelli, J. L., St. John, H. K., Cho, E. \& Suchman, N. E. (2016). Reflective functioning in parents of school-aged children. American Journal of Orthopsychiatry, 86, 24-36. https://doi.org/10.1037/ort0000141

Campbell, L. \& Kashy, D. A. (2002). Estimating actor, partner, and interaction effects for dyadic data using PROC MIXED and HLM: A user-friendly guide. Personal Relationships, 9, 327-342. https://doi.org/10.1111/1475-6811.00023

Castellano, R., Velotti, P., Crowell, J. A. \& Zavattini, G. C. (2014). The role of parents' attachment configurations at childbirth on marital satisfaction and conflict strategies. Journal of Child and Family Studies, 23, 1011-1026. https://doi.org/10.1007/s10826-013-9757-7

Chile, Ministerio de Salud (2011). Encuesta Nacional de Salud, ENS 2009-2010. Santiago, Chile: Autor.

Chile, Ministerio de Salud (2013). Guía clínica AUGE: depresión en personas de 15 años y más (2a ed.). Santiago, Chile: Autor, Subsecretaría de Salud Pública. 
Christopher, C., Umemura, T., Mann, T., Jacobvitz, D. \& Hazen, N. (2015). Marital quality over the transition to parenthood as a predictor of coparenting. Journal of Child and Family Studies, 24, 3636-3651. https://doi.org/10.1007/s10826-015-0172-0

Cox, M. J. \& Paley, B. (2003). Understanding families as systems. Current Directions in Psychological Science, 12, $193-196$. https://doi/org/10.1111/1467-8721.01259

Davies, P. T., Coe, J. L., Martin, M. J., Sturge-Apple, M. L. \& Cummings, E. M. (2015). The developmental costs and benefits of children's involvement in interparental conflict. Developmental Psychology, 51, 1026-1047. https://doi/org/10.1037/dev0000024

Davila, J., Karney, B. R., Hall, T. W. \& Bradbury, T. N. (2003). Depressive symptoms and marital satisfaction: Within-subject associations and the moderating effects of gender and neuroticism. Journal of Family Psychology, 17, 557-570. https://doi/org/10.1037/0893-3200.17.4.557

Dinkel, A. \& Balck, F. (2005). An evaluation of the German Relationship Assessment Scale. Swiss Journal of Psychology, 64, $259-263$. https://doi.org/10.1024/1421-0185.64.4.259

Ensink, K., Bégin, M., Normandin, L. \& Fonagy, P. (2016). Maternal and child reflective functioning in the context of child sexual abuse: Pathways to depression and externalising difficulties. European Journal of Psychotraumatology, 7, artículo 30611. https://doi.org/10.3402/ejpt.v7.30611

Ensink, K., Bégin, M., Normandin, L. \& Fonagy, P. (2017). Parental reflective functioning as a moderator of child internalizing difficulties in the context of child sexual abuse. Psychiatry Research, 257, 361-366. https://doi.org/10.1016/j.psychres.2017.07.051

Faulkner, R. A., Davey, M. \& Davey, A. (2005). Gender-related predictors of change in marital satisfaction and marital conflict. The American Journal of Family Therapy, 33, 61-83. https://doi.org/10.1080/01926180590889211

Favez, N., Lopes, F., Bernard, M., Frascarolo, F., Lavanchy Scaiola, C., Corboz- Warnery, A. \& Fivaz-Depeursinge, E. (2012). The development of family alliance from pregnancy to toddlerhood and child outcomes at 5 years. Family Process, 51, 542-556. https://doi.org/10.1111/j.1545-5300.2012.01419.x

Fincham, F. D., Beach, S. R. H., Harold, G. T. \& Osborne, L. N. (1997). Marital satisfaction and depression: Different causal relationships for men and women? Psychological Science, 8, 351-356. https://doi.org/10.1111/j.1467-9280.1997.tb00424.x

Fischer-Kern, M., Fonagy, P., Kapusta, N. D., Luyten, P., Boss, S., Naderer, A. ... Leithner, K. (2013). Mentalizing in female inpatients with major depressive disorder. The Journal of Nervous and Mental Disease, 201, 202-207. https://doi.org/10.1097/NMD.0b013e3182845c0a

Fonagy, P., Gergely, G., Jurist, E. \& Target, M. (2004). Affect regulation, mentalization and the development of the self ( $2^{\mathrm{a}}$ ed.). London Reino Unido: Karnac Books.

Fonagy, P., Steele, M., Steele, H., Moran, G. S. \& Higgitt, A. C. (1991). The capacity for understanding mental states: The reflective self in parent and child and its significance for security of attachment. Infant Mental Health Journal, 12, $201-218$. https://doi.org/10.1002/1097-0355(199123)12:3<201::AID-IMHJ2280120307>3.0.CO;2-7

Fonagy, P., Target, M., Steele, H. \& Steele, M. (1998). Reflective-functioning manual, version 5.0, for application to adult attachment interviews. London, Reino Unido: University College London.

Gabriel, B., Beach, S. R. H. \& Bodenmann, G. (2010). Depression, marital satisfaction and communication in couples: Investigating gender differences. Behavior Therapy, 41, 306-316. https://doi.org/10.1016/j.beth.2009.09.001

Goodman, J. H. (2008). Influences of maternal postpartum depression on fathers and on father-infant interaction. Infant Mental Health Journal, 29, 624-643. https://doi.org/10.1002/imhj.20199

Gottman, J. (1999). The marriage clinic: A scientifically based marital therapy. New York, NY: Norton.

Hayes, A. F. (2013). Introduction to mediation, moderation, and conditional process analysis: A regression-based approach. New York, NY: Guilford Press.

Heene, E., Buysse, A. \& Van Oost, P. (2007). An interpersonal perspective on depression: The role of marital adjustment, conflict communication, attributions, and attachment within a clinical sample. Family Process, 46, 499-514. https://doi.org/10.1111/j.15455300.2007.00228.x

Hendrick, S. S. (1988). A generic measure of relationship satisfaction. Journal of Marriage and Family, 50, 93-98. https://doi.org/10.2307/352430

Hughes, J. (2017). reghelper: Helper functions for regression analysis. $R$ package version 0.3.3. Wien, Austria: The R Foundation for Statistical Computing. Extraído de https://CRAN.R-project.org/package=reghelper

Hyde, J. S., Mezulis, A. H. \& Abramson, L. Y. (2008). The ABCs of depression: Integrating affective, biological, and cognitive models to explain the emergence of the gender difference in depression. Psychological Review, 115, 291-313. https://doi.org/10.1037/0033-295X.115.2.291

Kenny, D. A., Kashy, D. A. \& Cook, W. L. (2006). Dyadic data analysis. New York, NY: Guilford Press.

Kouros, C. D., Papp, L. M. \& Cummings, E. M. (2008). Interrelations and moderators of longitudinal links between marital satisfaction and depressive symptoms among couples in established relationships. Journal of Family Psychology, 22, 667-677. https://doi.org/10.1037/0893-3200.22.5.667

Ladegaard, N., Lysaker, P. H., Larsen, E. R. \& Videbech, P. (2014). A comparison of capacities for social cognition and metacognition in first episode and prolonged depression. Psychiatry Research, 220, 883-889. https://doi.org/10.1016/j.psychres.2014.10.005

Lefcheck, J. S. (2016). PIEcewiseSEM: Piecewise structural equation modeling in R for ecology, evolution, and systematics. Methods in Ecology and Evolution, 7, 573-579. https://doi.org/10.1111/2041-210X.12512

Mattern, M., Walter, H., Hentze, C., Schramm, E., Drost, S., Schoepf, D. ... Schnell, K. (2015). Behavioral evidence for an impairment of affective theory of mind capabilities in chronic depression. Psychopathology, 48, 240-250. https://doi.org/10.1159/000430450

Nakagawa, S. \& Schielzeth, H. (2013). A general and simple method for obtaining $R^{2}$ from generalized linear mixed- effects models. Methods in Ecology and Evolution, 4, 133-142. https://doi.org/10.1111/j.2041-210x.2012.00261.x

Paulson, J. F. \& Bazemore, S. D. (2010). Prenatal and postpartum depression in fathers and its association with maternal depression: A meta-analysis. JAMA: The Journal of the American Medical Association, 303, 1961-1969. https://doi.org/10.1001/jama.2010.605

Peugh, J. L. (2010). A practical guide to multilevel modeling. Journal of School Psychology, 48, 85-112. https://doi.org/10.1016/j.jsp.2009.09.002

Piccinelli, M. \& Wilkinson, G. (2000). Gender differences in depression: Critical review. The British Journal of Psychiatry, $177,486-492$. https://doi.org/10.1192/bjp.177.6.486

Pinheiro, J., Bates, D., DebRoy, S., Sarkar, D. \& R Core Team (2017). nlme: Linear and nonlinear mixed effects models. $R$ package version 3.1-131. Wien, Austria: R Foundation for Statistical Computing. Extraído de https://CRAN.R-project.org/package=nlme

Preacher, K. J., Curran, P. J. \& Bauer, D. J. (2006). Computational tools for probing interactions in multiple linear regression, multilevel modeling, and latent curve analysis. Journal of Educational and Behavioral Statistics, 31, 437-448. https://doi.org/10.3102/10769986031004437

$\mathrm{R}$ Development Core Team (2011). R: A language and environment for statistical computing. Wien, Austria: R Foundation for Statistical Computing. Extraído de http://www.R-project.org 
Rivera, D., Cruz, C. \& Muñoz, C. (2011). Satisfacción en las relaciones de pareja en la adultez emergente: el rol del apego, la intimidad y la depresión. Terapia Psicológica, 29, 77-83. https://doi.org/10.4067/S0718-48082011000100008

Sethna, V., Murray, L. \& Ramchandani, P. G. (2012). Depressed fathers' speech to their 3-month-old infants: A study of cognitive and mentalizing features in paternal speech. Psychological Medicine, 42, 2361-2371. https://doi.org/10.1017/S0033291712000487

Shapiro, A. F., Gottman, J. M. \& Carrère, S. (2000). The baby and the marriage: Identifying factors that buffer against decline in marital satisfaction after the first baby arrives. Journal of Family Psychology, 14, 59-70. https://doi.org/10.1037/0893-3200.14.1.59

Shieh, G. (2011). Clarifying the role of mean centring in multicollinearity of interaction effects. British Journal of Mathematical and Statistical Psychology, 64, 462-477. https://doi.org/10.1111/j.2044-8317.2010.02002.x

Slade, A. (2005). Parental reflective functioning: An introduction. Attachment \& Human Development, 7 , $269-281$. https://doi.org/10.1080/14616730500245906

Slade, A., Aber, J. L., Berger, B., Bresgi, I. \& Kaplan, M. (2012). The parent development interview-revised, short version. Manuscrito no publicado, The City University of New York, NY, Estados Unidos.

Slade, A., Bernbach, E., Grienenberger, J., Levy, D. \& Locker, A. (2005). Addendum to Fonagy, Target, Steele, \& Steele reflective functioning scoring manual for use with the Parent Development Interview, version 2.0. Manuscrito no publicado, The City College and Graduate Center of the City University of New York, NY, Estados Unidos.

Slade, A., Grienenberger, J., Bernbach, E., Levy, D. \& Locker, A. (2005). Maternal reflective functioning, attachment, and the transmission gap: A preliminary study. Attachment \& Human Development, 7, 283-298. https://doi.org/10.1080/14616730500245880

Sleed, M., Baradon, T. \& Fonagy, P. (2013). New beginnings for mothers and babies in prison: A cluster randomized controlled trial. Attachment \& Human Development, 15, 349-367. https://doi.org/10.1080/14616734.2013.782651

Squires, J., Bricker, D. \& Twombly, E. (2002). Ages \& Stages Questionnaires: Social-Emotional. A parent-completed child-monitoring system for social-emotional behaviors. Baltimore, MD: Paul H. Brookes.

Steele, H. \& Steele, M. (2008). On the origins of reflective functioning. En F. N. Busch (Ed.), Mentalization: Theoretical considerations, research findings, and clinical implications (pp.133-158). New York, NY: Analytic Press.

Suchman, N. E., Decoste, C., Rosenberger, P. \& McMahon, T. J. (2012). Attachment-based intervention for substance-using mothers: A preliminary test of the proposed mechanisms of change. Infant Mental Health Journal, 33, 360-371. https://doi.org/10.1002/imhj.21311

Tapia Villanueva, L., Poulsen, G., Armijo, I., Pereira, X. \& Sotomayor, P. (2009). Resolución de entrampes en parejas en conflicto: aproximaciones desde las parejas y los terapeutas. Revista Argentina de Clínica Psicológica, 18, 101-114. Extraído de https://dialnet.unirioja.es/servlet/articulo?codigo $=4266032$

Tissot, H., Favez, N., Ghisletta, P., Frascarolo, F. \& Despland, J. -N. (2017). A longitudinal study of parental depressive symptoms and coparenting in the first 18 months. Family Process, 56, 445-458. https://doi.org/10.1111/famp.12213

Turner, J. M., Wittkowski, A. \& Hare, D. J. (2008). The relationship of maternal mentalization and executive functioning to maternal recognition of infant cues and bonding. British Journal of Psychology, 99, 499-512. https://doi.org/10.1348/000712608X289971

Uekermann, J., Channon, S., Lehmkämper, C., Abdel-Hamid, M., Vollmoeller, W. \& Daum, I. (2008). Executive function, mentalizing and humor in major depression. JINS: Journal of the International Neuropsychological Society, 14, 55-62. https://doi.org/10.1017/S1355617708080016

Valdés, C., Morales-Reyes, I., Pérez, J. C., Medellín, A., Rojas, G. \& Krause, M. (2017). Propiedades psicométricas del Inventario de Depresión de Beck IA para la población chilena. Revista Médica de Chile, 145, 1005-1012. https://doi.org/10.4067/s0034-98872017000801005

Wee, K. Y., Skouteris, H., Pier, C., Richardson, B. \& Milgrom, J. (2011). Correlates of ante- and postnatal depression in fathers: A systematic review. Journal of Affective Disorders, 130, 358-377. https://doi.org/10.1016/j.jad.2010.06.019

Wickham, H. (2002) ggplot2: Elegant graphics for data analysis. New York, NY: Springer-Verlag.

Wong, K. (2012). A study of the effect of maternal depressive symptoms on the mother-infant relationship and protective effect of maternal reflective functioning (Tesis de Maestría, Wayne State University, Detroit, MI, Estados Unidos). Extraído de https://digitalcommons.wayne.edu/ cgi/viewcontent.cgi?article=1257\&context=0a_theses

Fecha de recepción: Diciembre de 2016.

Fecha de aceptación: Abril de 2018. 